\title{
DOCENCIA UNIVERSITARIA DURANTE LA PANDEMIA COVID-
} 19: UNA MIRADA DESDE CHILE

Felipe Andres Zurita Garrido ${ }^{1}$

\section{RESUMEN}

El presente artículo tiene como objetivo presentar un relato libre sobre una experiencia personal de docencia universitaria durante la pandemia COVID-19 en el contexto del sistema universitario de Chile. Desde el punto de vista metodológico, el trabajo se construyó en base a la articulación de un relato basado en la memoria y experiencia personal del autor, lo que permitió articular una argumentación estructurada en diversas reflexiones abiertas sobre el ser docente universitario en el contexto de emergencia sanitaria. Los resultados de esta reflexión se articularon a través de las categorías de análisis de tiempo, tecnología y relación pedagógica. A modo de conclusión se comparten algunas reflexiones basadas en interrogantes y reflexiones que emergieron a partir de lo narrado y que se presentan como una oportunidad de articulación de nuevas reflexiones posibles.

Palabras clave: Docencia universitaria. Pandemia COVID-19. Chile.

\section{Como citar este documento - ABNT}

GARRIDO, Felipe Andres Zurita. Docencia universitaria durante la pandemia COVID-19: una mirada desde Chile. Revista Docência do Ensino Superior, Belo Horizonte, v. 10, e024777, p. 1-9, 2020. DOI: https://doi.org/10.35699/22375864.2020.24777.
Recebido em: 19/08/2020

Aprovado em: $16 / 10 / 2020$ Publicado em: $14 / 11 / 2020$

\footnotetext{
${ }^{1}$ Universidad Metropolitana de Ciencias de la Educación (UMCE), Ñuñoa, Chile. ORCID ID: https://orcid.org/0000-0002-4136-4340. E-mail: felipe.zurita@umce.cl.
} 


\section{DOCÊNCIA UNIVERSITÁRIA DURANTE A PANDEMIA DA COVID-19: UM OLHAR DO CHILE}

\section{RESUMO}

O presente artigo tem como objetivo apresentar um relato livre sobre uma experiência pessoal de docência universitária durante a pandemia da COVID-19 no contexto do sistema universitário chileno. Do ponto de vista metodológico, o trabalho foi construído a partir da articulação de um relato baseado na memória e da experiência pessoal do autor, o que permitiu articular um argumento estruturado em várias reflexões abertas sobre ser professor universitário no contexto de emergência sanitária. Os resultados dessa reflexão foram articulados por meio das categorias de análise de tempo, tecnologia e relação pedagógica. A título de conclusão, são compartilhadas algumas reflexões baseadas em questionamentos e reflexões que emergiram da narrativa e que se apresentam como uma oportunidade de articulação de novas reflexões possíveis.

Palavras-chave: Docência universitária. Pandemia da COVID-19. Chile.

\section{UNIVERSITY TEACHING DURING THE COVID-19 PANDEMIC: A VIEW FROM CHILE}

\section{ABSTRACT}

This article aims to present a free report on a personal experience of university teaching during the COVID-19 pandemic in the context of the Chilean university system. From a methodological point of view, the work was built from the articulation of a report based on the author's memory and personal experience, which allowed articulating a structured argumentation in several open reflections on being a university professor in the context of health emergencies. The results of this reflection were articulated through the categories of analysis of time, technology and pedagogical relationship. As a conclusion, some reflections are shared based on questions and reflections that emerged from the narrated and that present themselves as an opportunity to articulate new possible reflections.

Keywords: University teaching. COVID-19 pandemic. Chile. 


\section{INTRODUCCIÓN}

La pandemia COVID-19 ha cubierto de manera rápida y poderosa a la humanidad entera con su manto de muerte e incertidumbre, obligando a replantear aspectos fundamentales de la vida contemporánea. Chile se encuentra entre aquellos países más afectados del continente americano, de hecho, al momento de escribir estas líneas se alcanzan las dolorosas cifras de más de 380.000 personas infectadas y más de 10.000 personas fallecidas confirmadas por COVID-19 (CHILE, 2020).

En el contexto anterior, a pesar de estar desarrollándose la pandemia COVID-19, en Chile durante todo el año 2020 se han desarrollado actividades de docencia en el sistema universitario en la modalidad no presencial. Lo anterior obligó a que miles de docentes y estudiantes tuviesen que desarrollar sus actividades en una modalidad desconocida, a través de plataformas digitales en los casos en que el acceso a dispositivos y conexión a internet lo permitiera.

En este escrito, se presentarán algunas reflexiones que buscan comunicar mi experiencia personal en tanto profesor universitario en el contexto de la pandemia COVID-19 en Chile. Para realizar lo anterior, he organizado mis reflexiones en los siguientes apartados: primero, comunicaré algunas informaciones básicas para poder comprender la estructura del sistema universitario chileno a modo de contextualización sobre en qué contexto las y los docentes desarrollamos nuestras actividades de docencia; segundo, resaltaré algunas reflexiones sobre los aspectos más relevantes de mi experiencia como profesor universitario en la modalidad no presencial; tercero, a modo de cierre compartiré algunas reflexiones sobre cuáles son las proyecciones y preocupaciones que la experiencia de docencia universitaria en la modalidad no presencial me han permitido construir.

\section{DOCENCIA UNIVERSITARIA EN CHILE DURANTE LA PANDEMIA COVID-19}

El sistema universitario chileno contemporáneo se caracteriza por su alto nivel de privatización y mercantilización. Privatización debido a que de un total de 59 universidades que conforman el sistema, 41 son privadas y solamente 18 son estatales (CHILE, 2019). Mercantilización debido a que por décadas el sistema universitario ha funcionado con regulaciones flexibles que han permitido que una parte de él pudiese orientar sus actividades detrás de la acumulación de capital como objetivo prioritario, especialmente aquellas universidades privadas pertenecientes a grupos empresariales nacionales y trasnacionales (MÖNCKEBERG, 2007, 2013). Para que esto fuese posible es importante señalar que todo el sistema universitario se financia de forma mayoritaria en base al cobro de matrículas $y$ aranceles a las y los estudiantes a un costo muy elevado para la realidad local, lo que es asumido por las y los estudiantes a través del pago directo o a través de sistemas de 
financiamiento en base a créditos a largo plazo que han generado un alto nivel de endeudamiento entre la población (PÁEZ; KREMERMAN, 2016).

Esta estructura del sistema universitario caracterizada por ser privatizada y mercantilizada fue configurada durante la Dictadura Cívico Militar (1973-1990) donde se interrumpió la extensa tradición del sistema universitario chileno de carácter público y gratuito al establecer por medio de la fuerza el arancelamiento, la desestructuración de las universidades estatales y la flexibilización de la regulación para favorecer la creación de universidades privadas. Dicha estructuración privatizada y mercantilizada fue protegida y fortalecida por parte de los Gobierno de la Transición a la Democracia, a través de regulaciones que favorecieron la masificación del acceso a la universidad en base al endeudamiento y a la libertad de acción de las universidades orientadas a la obtención de altísimos lucros.

El escenario anteriormente señalado sólo comenzó a ser cuestionado a partir de las extensas y masivas movilizaciones de las y los estudiantes secundarios y universitarios a inicios del siglo $\mathrm{XXI}$, que instalaron en el debate público la necesidad de reorganizar el sistema educacional en todos sus niveles en base a los principios de fortalecimiento de lo público, gratuidad y calidad.

¿Qué espacio tienen las y los docentes en este sistema universitario caracterizado por la privatización y la mercantilización? Las y los profesionales que realizan actividades de docencia en el sistema universitario chileno desarrollan sus actividades en una condición de precariedad y flexibilidad notorias. Durante la pandemia COVID-19 esta condición se agudizó, puesto que el sistema universitario comenzó a vivir dificultades de financiamiento debido a que sectores importantes de las y los estudiantes dejaron de pagar sus estudios o los congelaron, generando así una disminución en el ingreso de recursos de las universidades. En este escenario, en algunas universidades se procedió a desvincular a docentes, especialmente a aquellos que tenían un vínculo laboral más frágil.

\section{EXPERIENCIA}

Durante el primer semestre 2020 trabajé como docente en dos universidades privadas en la modalidad de profesor investigador contratado ${ }^{2}$ y me correspondió realizar actividades de docencia a varios grupos de estudiantes en la modalidad no presencial, en cursos de formación pedagógica de las áreas de Historia de la Educación, Didáctica de la Historia e Investigación Educacional. En este apartado describiré mi experiencia como docente universitario en el contexto de la pandemia COVID-19 organizando mis argumentos en relación a las nociones de tiempo, tecnología y relación pedagógica.

\footnotetext{
${ }^{2}$ Actualmente trabajo en una universidad estatal a la que accedí recientemente mediante concurso público.
} 


\section{Tiempo}

Durante el primer semestre 2020 realicé aproximadamente 17 horas cronológicas de clases semanales. Este número de horas de clases no es habitual en mi experiencia como profesor universitario y se vio aumentada debido a que en las universidades en que trabajaba se realizaron ajustes que implicaban que las y los docentes contratados tuvimos que realizar más horas de docencia que lo pactado tradicionalmente, como estrategia para hacer frente al escenario de crisis económica que se abría frente la pandemia COVID-19. De esta forma, tuve que afrontar un ciclo con un inédito alto número de horas de docencia, precisamente en un contexto de profundas transformaciones en el trabajo de enseñar.

De manera evidente, más allá de lo mencionado con anterioridad que corresponde a una situación personal, la modalidad de docencia a distancia implica que las horas dedicadas al trabajo de docencia se incrementen de manera radical. Las actividades tradicionales de preparación de la docencia, diálogo pedagógico y retroalimentación de las actividades se tornaron claramente más complejas y difíciles de enfrentar.

En el caso de la preparación de la docencia, más allá del trabajo de preparación de lecturas y materiales de enseñanza, se sumó: la obligación de completar extensas y complejas plataformas digitales con información de cada uno de los cursos; la necesidad de generar y distribuir invitaciones para acceder a la plataforma de conexión sincrónica correspondiente (Zoom o Teams); grabar las clases y dejarlas disponible en la plataforma de Youtube que quedasen a disposición de las y los estudiantes que no podían conectarse a ellas; entre otras.

Con respecto al diálogo pedagógico, creo que se trata de un factor crítico, puesto que en la modalidad a distancia mediada por tecnologías la comunicación se hace compleja y entrecortada. Aquellas cuestiones que se resolvían de manera sencilla en el contexto de clases presenciales, tales como atender dudas o dificultades para comprender alguna indicación, en la modalidad de clases a distancia se complejizan debido a que nada de lo que se indique debe ser asumido como dicho, ya que no hay claridad con cuántos estudiantes se está trabajando. De esta forma, las explicaciones, orientaciones, analogías, recordatorios, sugerencias, etc., que tradicionalmente realizo, muchas veces se terminaron tornado superfluas, ya que debía repetirlas constantemente, tomando mucho más tiempo del habitual.

Finalmente, en el caso de la retroalimentación de las actividades de evaluación, el tiempo de dedicación se amplió de manera abierta, puesto que frente a la imposibilidad de concretar una retroalimentación en base al diálogo directo y sencillo, tuve que optar por desarrollar la corrección y escritura de sugerencias en los diferentes trabajos en formato digital de cada estudiante, los que muchas veces eran insuficientes y abrían espacio a nuevas dudas que debían atenderse en múltiples correos electrónicos colectivos o individuales. Esto abrió paso, en contados casos, a la posibilidad de desarrollar diálogos interesantes y ricos en significados 
con estudiantes, incluso más allá de los temas propiamente vinculados a la evaluación o trabajo específico, a modo de desarrollo de conversaciones paralelas sobre el curso o la vida en un contexto de pandemia.

\section{Tecnología}

La modalidad de educación a distancia mediada por tecnología me presentó un tremendo desafío de aprendizaje y organización, puesto que en general mi conocimiento en torno al uso de tecnologías para la enseñanza era bastante limitado. Para poder afrontar mis actividades de docencia tuve que aprender a usar plataformas para realizar clases online, fundamentalmente Zoom y Teams, las que me permitieron encontrarme semanalmente con las y los estudiantes que estaban en condiciones de conectarse a través de sus celulares y computadores. Para poder generar condiciones de llegar a todas y todos los estudiantes, incluso quienes no tenían las condiciones de contactarse con regularidad debido a problemas de conectividad u otros, tuve que abrir un canal de Youtube para compartir las grabaciones de mis clases y dejarlas disponibles en las plataformas digitales Moodle de cada universidad. Para poder intercambiar documentos y construir trabajos de manera colectiva aprendí a utilizar Google Drive. Para poder recoger información estandarizada de manera rápida y sencilla aprendí a utilizar Google Forms.

Más allá del proceso de aprendizaje sobre el uso de las plataformas mencionadas con anterioridad, que es de carácter básico, creo relevante mencionar un par de reflexiones en torno a la tecnología con fines educacionales. En primer lugar, es importante reconocer la existencia de múltiples herramientas tecnológicas que pueden constituirse en oportunidades de ampliación de las oportunidades de aprendizaje de las y los estudiantes. Esto, desde mi punto de vista de docente universitario, me parece que es importante de ser reconocido y explorado por nuestra parte, asumiendo de esta forma que es parte de las responsabilidades pedagógicas buscar todas las alternativas posibles para favorecer el aprendizaje de las y los estudiantes universitarios.

Lo anteriormente señalado, también me obliga a plantear una segunda reflexión, en la línea de que en la modalidad de clases a distancia mediada por tecnologías el aprendizaje de las y los estudiantes también necesita de la disposición y la curiosidad de parte de las y los mismos estudiantes. Más allá de lo que puedan pensar y defender los optimistas tecnológicos, creo que la tecnología no construye finalmente un mundo pedagógico nuevo, detrás de las pantallas y las luces más o menos coloridas están las y los mismos actores y los mismos objetivos: profesoras, profesores y estudiantes organizados en base a la promesa de enseñar y aprender mutuamente algunos conocimientos, experimentar algunas habilidades y sensibilizarse con algunas actitudes. Por cierto, que en el contexto de pandemia COVID-19 estos objetivos y promesas adquirieron tonos diferentes y elementos urgentes, aunque profundamente humanos, tales como simplemente encontrarse y hablar. 
Una tercera reflexión con la tecnología corresponde al sentido de su uso. Desgraciadamente en algunos casos las tecnologías fueron entendidas como una perfecta extensión para los afanes burocráticos que envuelven las actividades de docencia universitaria. En esta línea, no es de extrañar que se asumiera con naturalidad la necesidad de imponer a las y los docentes la obligación de alimentar de información diferentes plataformas, con el objetivo de dejar evidencia de lo obrado. Esto es interesante y necesario de atender: no basta con cumplir con la preparación y la realización de las clases, era necesario generar registros múltiples de lo realizado, los que se tornaron críticamente obligatorios y urgentes de atender. Este tipo de uso de la tecnología seguramente instala el sinsentido como un elemento que intermedia las actividades de las y los docentes con relación a la docencia.

Una cuarta y última reflexión sobre la tecnología y su relación con la docencia en contextos de pandemia, tiene que ver con que a través de las mismas se facilitó la imposición de un nuevo tipo de relación laboral, donde se asume que las y los docentes están disponibles en todo momento para atender a estudiantes y a las jefaturas directas, mostrando una faceta compleja del teletrabajo que favorece que el tiempo y el espacio íntimo de las y los docentes se desdibuje.

\section{Relación Pedagógica}

En mi experiencia de docencia universitaria en la modalidad a distancia mediada por tecnologías puedo señalar que viví un tipo de relación pedagógica basada en la incertidumbre. Nunca sentí seguridad en torno a cuestiones básicas de la relación pedagógica, tales como quiénes eran (desde una perspectiva profunda) las y los estudiantes con los que trabajé, o si acaso las y los estudiantes me escuchaban al otro lado de la pantalla. La imagen de Zoom colmada de cuadros negros y silenciosos resultó ser algo familiar en mis actividades diarias, donde lo que veía con frecuencia era mi propia imagen reflejada en la pantalla. Opté por entender que esto tenía que ver con una estrategia de las y los estudiantes de protección de su espacio íntimo y lo respeté sin dificultad. La incertidumbre también corrió por la línea de la pregunta en torno a si las y los estudiantes estaban aprendiendo lo programado en cada uno de los cursos que realicé. En este caso la incertidumbre tuvo que ver con que finalmente el momento que estábamos viviendo correspondía a una profunda crisis multidimensional que finalmente nos invitaba a las y los docentes a replantearnos la orientación y el sentido de cada uno de nuestros cursos y exigía de nosotros realizar propuestas formativas lo suficientemente flexibles y contextualizadas que favorecieran trabajar aquellos aspectos fundamentales de cada asignatura junto a aquellos elementos emergentes y urgentes que íbamos viviendo. Las nociones de comprensión y empatía fueron ganando un espacio privilegiado en el lenguaje cotidiano, lo que fue facilitando el desarrollo de las múltiples actividades comprometidas en la medida de las posibilidades existentes. No tengo una respuesta contundente sobre qué o cuánto aprendieron las y los estudiantes de cada uno de mis asignaturas. Creo que las y los 
profesores ahora menos que nunca podemos responder esto, especialmente porque varias y varios estudiantes nunca establecieron cualquier tipo de interacción o contacto en algunos de estas, ya sea porque tenían graves problemas de conectividad o porque estaban viviendo graves situaciones sanitarias o económicas en su círculo más cercano o en su propia piel. Más allá de los acotados e insuficientes esfuerzos que realizaron las universidades por proveer de equipos computacionales o bolsas prepago de internet, queda esa sensación de que finalmente las y los estudiantes fueron abandonados a su suerte en torno a si se conectaban o no a las clases por las que estaban pagando.

\section{CONSIDERACIONES FINALES}

Al cerrar la presentación de esta experiencia quedo con más dudas que seguridad. ¿Valió la pena haber desarrollado clases universitarias en el contexto de la pandemia COVID-19 o era mejor haber hecho una pausa en los procesos de formación profesional para concentrarse en proteger la salud física y mental de toda la comunidad? ¿La modalidad de educación a distancia mediada por tecnologías es apropiada para la formación inicial de profesoras y profesores? ¿Qué transformaciones de las experimentadas en este tiempo se instalarán en las universidades y qué consecuencias estas tendrán para la docencia universitaria?

En el caso del sistema universitario chileno, marcado por las características ya señaladas, la pausa esbozada en la primera pregunta nunca fue una posibilidad, fundamentalmente debido a que desde la lógica de financiamiento privado instalada como hegemónica la "venta" y "consumo" de servicios educacionales no puede parar. Como no puede parar, independiente del tiempo, el espacio y la vida concreta, se fuerza su funcionamiento.

Con respecto a la segunda pregunta, me parece que el daño que sufre la relación pedagógica en la modalidad de docencia no presencial mediada por tecnologías es tan grande, que pone en entredicho la proyección de ella en el tiempo para la formación inicial docente más allá de este escenario de emergencia. Vinculando esto con la tercera interrogante, creo que existen incentivos económicos poderosos para instalar con fuerza esta modalidad en la docencia universitaria, lo que nos invita a reflexionar seriamente sobre las consecuencias de su potencial extensión, especialmente sobre aquella que fragiliza y hace superficial la relación pedagógica entre docentes y estudiantes.

\section{REFERENCIAS}

CHILE. Subsecretaria de Educación Superior. Informe Personal Académico en Educación Superior 2019, Santiago: Servicio de Información de Educación Superior, noviembre de 2019.

CHILE. Ministerio de Salud. Informe Epidemiológico № 42 Enfermedad por SARS-CoV-2 (COVID-19), Santiago: Departamento de Epidemiologia, 14 de agosto de 2020. 
MÖNCKEBERG, María Olivia. El negocio de las universidades en Chile. Santiago: Debate, 2007.

MÖNCKEBERG, María Olivia. Con fines de lucro: la escandalosa historia de las universidades privadas en Chile. Santiago: Random House Mondadori, 2013.

PÁEZ, Alexander; KREMERMAN, Marco. Endeudar para gobernar y mercantilizar: el caso del CAE. Santiago: Fundación Sol, 2016.

\section{Felipe Andres Zurita Garrido}

Profesor de Historia y Ciencias Sociales, Licenciado en Educación y Magíster en Educación, Mención Didáctica e Innovación Pedagógica (Universidad Academia de Humanismo Cristiano, Chile). Doctor en Educación (Universidad Federal de Minas Gerais, Brasil). Actualmente se desempeña como Profesor Investigador en el Departamento de Educación Básica de la Facultad de Filosofía y Educación de la Universidad Metropolitana de Ciencias de la Educación (UMCE), Chile.

felipe.zurita@umce.cl 